\title{
Role of Acupuncture and Fire Cupping in Reducing the Thyroxine Dose and Improving the Thyroid Function in Hypothyroidism Patients: A Case Series
}

\author{
Pradeep M. K. Nair ${ }^{1, *}$, Naga Jyothi ${ }^{2}$ \\ 1 Indian Academy for Scientific Writing and Research, Pune, India \\ ${ }^{2}$ Dr. Jyoti's Nature Cure Clinic, Bangalore, India
}

Received March 22, 2021

Revised August 5, 2021

Accepted August 17, 2021

Correspondence to Pradeep M. K. Nair

Indian Academy for Scientific Writing and Research, Pune, India

E-mail director@iaswar.com
Hypothyroidism is one of the most common endocrine disorders linked to various systemic diseases ranging from obesity to cancers. The present line of management is insufficient as reports suggest that there is persistence of symptoms and poor adherence that makes the management of said disease challenging. Acupuncture and other Traditional Chinese Medicine (TCM) therapies are proven to alleviate endocrine dysfunctions. However, reports on acupuncture and cupping therapy on hypothyroidism are very scarce. Here, we report 5 cases of hypothyroid patients aged between 38-44 yrs who were treated with acupuncture and fire cupping for a period of three months. At the baseline, the patients presented with either a weak, wiry or vacuum pulse which represents spleen Qi deficiency. Further, they presented with elevated levels of Thyroid Stimulating Hormone (TSH), and higher Body Mass Index (BMI). Acupuncture treatment at ST36, LI4, SP6, and BL20 was given bilaterally whilst CV4, LR3, LR4, SP9, ST40, SP10, GV4, KI3, ST12, and SI17 were punctured unilaterally. At the end of the time period of three months, patients progressed to normal levels of TSH, reduction in BMI and had succeeded in tapering their medication doses. Supplementing this, the patients reported marked improvement in other symptoms like fatigue, hair loss, and cold feet post-treatment. The effects were consistent even during the three month follow-up period post-interventions. The results encourage the utilization of acupuncture and fire cupping in the management of hypothyroidism. However, large scale studies are warranted to strengthen this recommendation.

Keywords: Hypothyroidism, Thyroxine, Acupuncture, Thyroid function, Fire cupping, Case report

\section{INTRODUCTION}

Hypothyroidism is the second most common endocrine disorder in women which is reported to affect $4-5 \%$ of the global population [1]. Unlike the global scenario in India, the prevalence of hypothyroidism is $11 \%$ where most of the cases remain undetected or untreated, thus affecting the performance and economy of Indian population [2]. Hypothyroidism is associated with various lifestyle disorders like obesity, metabolic syndromes, cardiovascular diseases and other endocrine disorders. Clinically, hypothyroidism presents with lethargy, hoarseness of voice, weight gain, chronic constipation and intolerance to cold in most demographics. However, many cases remain asymptomatic and these symptoms vary with the age and sex of the patients [3]. Hormone replacement therapy (HRT) using levothyroxine remains the common mode of treatment; however there remain a lot of challenges in implementing HRT like compliance, appropriate dosing, drug interactions and most importantly co-morbidities associated with hypothyroidism [4]. Recent reports dictate that a surge in dissatisfaction has found its place with the hypothyroidism patients under standard care. This is mainly because the existing system of standard care and approaches are found to be insufficient to handle the rising challenges and complications among hypothyroidism patients [5].

These reports are indicative of introducing combination therapies as an adjuvant to complement the existing line of care. Complementary and alternative medicine (CAM) is reckoned to have a greater role to play in many medical conditions including thyroid dysfunctions [6]. Acupuncture is one of many common modes of Traditional Chinese 
Medicine (TCM) therapy utilized for up-regulating the energy balance in hormonal irregularities. Along the same lines, fire cupping therapy is a well-established mode of TCM therapy in which cups of varying size are applied to select skin points to elicit pressure with the help of either heat or suction. Adding to its therapeutic effects, it has also been reported to be a credible preventative measure [7]. Delving in, according to TCM, hypothyroidism is classified into five typesheart Yang deficiency, kidney Qi deficiency, kidney Yang deficiency, spleen Qi deficiency and spleen Yang deficiency $[8,9]$. Although theoretical literature forms a solid ground of overwhelming evidence to support the use of acupuncture in hypothyroidism, the lack of clinical studies in this domain inhibits acupuncture from finding its footing here $[10,11]$. We present 5 cases of hypothyroidism treated with acupuncture and fire cupping for a period of 3 months.

\section{CASE PRESENTATION}

This case series describes 5 patients who have been ailing from hypothyroidism for a period of 5 years and were treated at a CAM setting in Bangalore, India. All the participants signed a written consent to participate in this study. The diagnosis of these patients was performed by their treating endocrinologist and the underlying cause of hypothyroidism was not discovered by them. The acupuncture therapy, fire cupping and dietary advice were provided by a licensed Yoga \& Naturopathy physician with 20 years of experience in diagnosing and treating using the mode of TCM. The cases were treated for three months with 24 sessions of acupuncture spaced out to be 8 sessions per month. Similarly, 4 sessions of fire cupping were mandated [12] per month amounting to 12 sessions over the course of these three months. Fire cupping is a method by which a cotton ball soaked in surgical spirit is lit and immediately placed into a cupping cup and removed quickly as the cup is placed on the skin creating a vacuum $[12,13]$. We have used 7 cups, of which size number 2 cups were applied over the C7 vertebrae level, around the umbilicus, ST 25, Ren 6 and Ren 9, whereas size number 3 cups were used at Sp 9 and St 34. Fig. 1 portrays a fire cupping session in progress. Good prognosis in fire cupping treatment (a pinkish light discoloration or no discoloration from initial bluish or pinkish discoloration) was observed approximately after 1 month which was also reflected in subjective symptomatic improvements as reported by the patient. All the patients were advised to be on a balanced diet that included wholesome grains, seasonal fruits and vegetable salads. The detailed description of the acupuncture needling is tabulated as per Standards for Reporting Interventions in Clinical Trials of Acupuncture (STRICTA) guidelines in Table 1 . We evaluated the change in thyroid function at the baseline followed by the end of 1 month, 2-months, 3-months and at the end of the 6 th month ( 3 months post-treatment). The timeline of the events is depicted in Fig. 2.

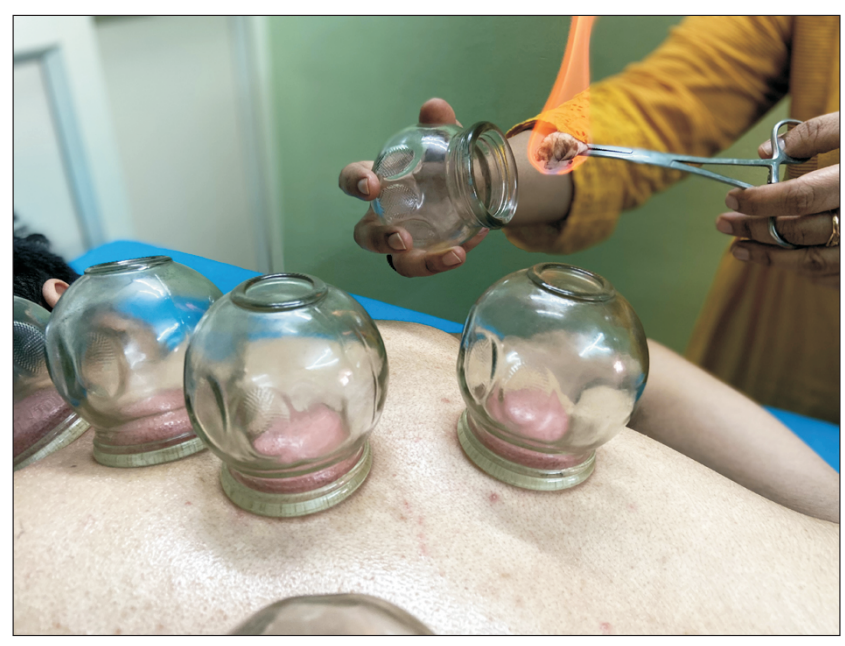

Fig. 1. A session of fire cupping in progress.

Table 1. Description of treatment regimen

\begin{tabular}{|c|c|c|}
\hline Style of acupuncture & \multicolumn{2}{|c|}{ Traditional Chinese acupuncture } \\
\hline Rationale for treatment & \multicolumn{2}{|c|}{ Spleen/kidney/Heart 'Yang' deficiency disorder } \\
\hline \multirow[t]{2}{*}{ Needling details: } & Bilateral needling points & ST36, LI4, SP6, and BL20 \\
\hline & Unilateral needling points & $\begin{array}{l}\text { CV4, LR3, LR4, SP9, ST40, SP10, GV4, } \\
\text { KI3, ST12, and SI17 }\end{array}$ \\
\hline Number of needles: & 19 needles & $\begin{array}{l}\text { Needle description } \\
\text { Needle type: Seirin }- \text { J type } \\
0.18 \mathrm{~mm} \times 15 \mathrm{~mm}(1 / 2 \text { tsun }) \\
0.18 \mathrm{~mm} \times 30 \mathrm{~mm}(1 \mathrm{tsun})\end{array}$ \\
\hline $\begin{array}{l}\text { Depth of insertion } \\
2-3 \mathrm{~cm} \text { in depth }\end{array}$ & $\begin{array}{l}\text { Response elicited } \\
\text { De Qi }\end{array}$ & $\begin{array}{l}\text { Needle stimulation method } \\
\text { Manual stimulation }\end{array}$ \\
\hline Needle retention time & & $\begin{array}{l}\text { - } 30 \text { minutes } \\
\text { - } 20 \text { minutes }\end{array}$ \\
\hline
\end{tabular}




\section{Case 1}

A 41-year-old woman with genetic predisposition to hypothyroidism was first diagnosed as hypothyroid during her pregnancy and was a known case of hypothyroidism for six years and was under the medication- Thyronorm (Thyroxine). She presented with elevated levels of Thyroid
Stimulating Hormone (TSH) and normal levels of Thyroxine (T4) and Triiodothyronine (T3) (Table 2). She has not reported any other symptoms except having a higher Body Mass Index (BMI). Evaluation of the tongue depicted a thin white coat with teeth marks that signifies spleen Qi deficiency and over thinking. Similarly, there was vacuity in the 2 nd

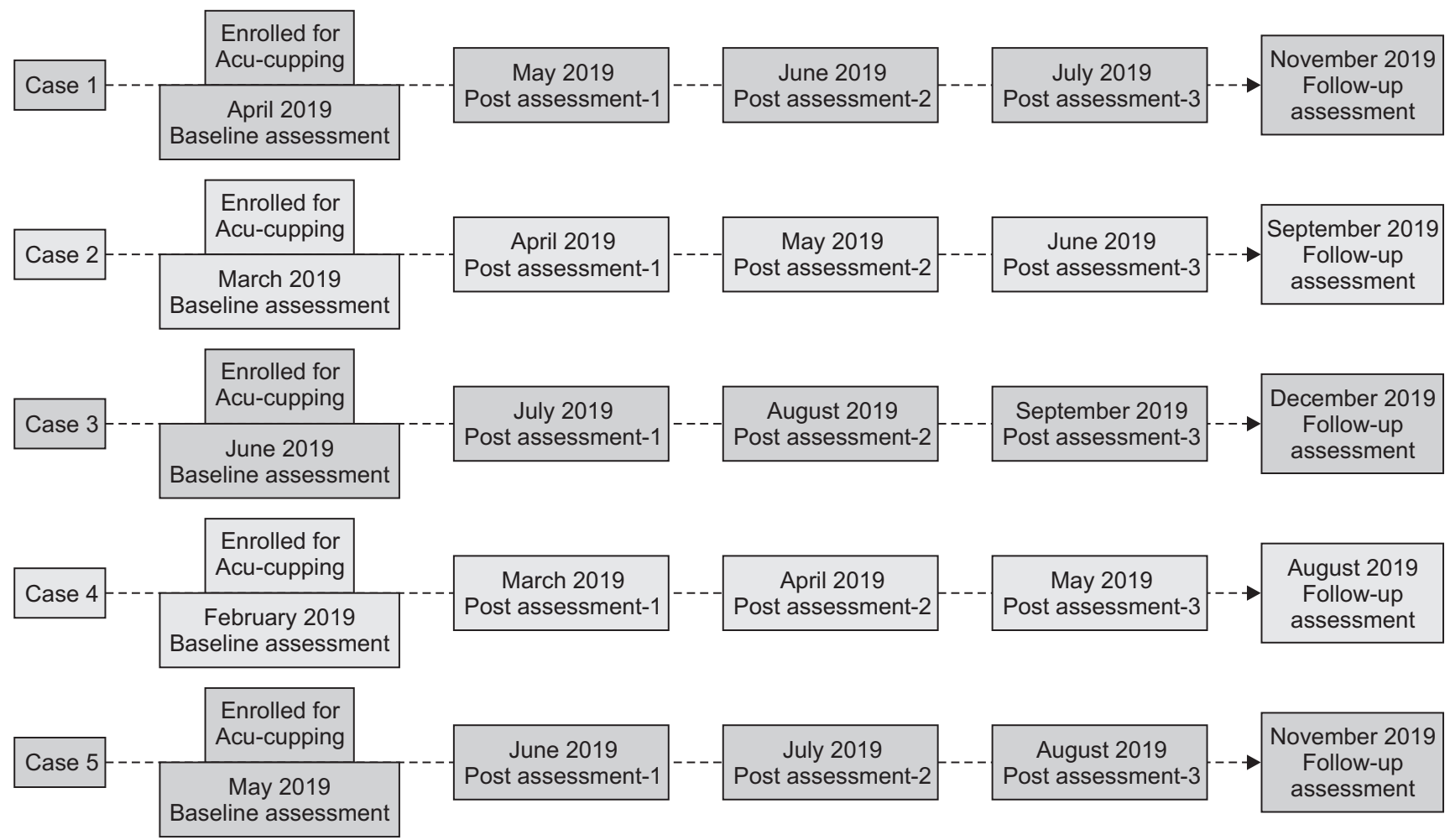

Fig. 2. Timeline of events.

Table 2. Changes in thyroid profile across different time points

\begin{tabular}{|c|c|c|c|c|c|}
\hline Cases & Thyroid Profile BL & Thyroid Profile M1 & Thyroid Profile M2 & Thyroid Profile M3 & Thyroid Profile M6 \\
\hline \multirow[t]{3}{*}{ Case 1} & T3-1.021 & $\mathrm{T} 3-1.310$ & T3-1.201 & T3-0.95 & T3-0.923 \\
\hline & $\mathrm{T} 4-8.740$ & $\mathrm{~T} 4-7.340$ & $\mathrm{~T} 4-7.830$ & $\mathrm{~T} 4-10.750$ & $\mathrm{~T} 4-10.620$ \\
\hline & TSH-14.2 & TSH-10.2 & TSH-6.2 & TSH-2.20 & $\mathrm{TSH}-3.20$ \\
\hline \multirow[t]{3}{*}{ Case 2} & T3-0.92 & T3-0.76 & T3-1.01 & T3-0.95 & T3-0.98 \\
\hline & $\mathrm{T} 4-3.52$ & $\mathrm{~T} 4-5.010$ & $\mathrm{~T} 4-8.010$ & $\mathrm{~T} 4-10.82$ & $\mathrm{~T} 4-10.720$ \\
\hline & TSH-11.32 & TSH-9.46 & TSH-6.600 & TSH-3.53 & TSH-3.43 \\
\hline \multirow[t]{3}{*}{ Case 3} & T3-0.82 & T3-0.67 & T3-1.012 & T3-0.91 & T3-0.913 \\
\hline & $\mathrm{T} 4-4.012$ & T4-6.101 & $\mathrm{T} 4-8.120$ & T4-10.520 & T4-10.630 \\
\hline & TSH-12.32 & TSH-8.021 & TSH-7.021 & TSH-3.32 & TSH-3.54 \\
\hline \multirow[t]{3}{*}{ Case 4} & T3-0.92 & T3-0.72 & T3-1.052 & T3-0.97 & T3-0.930 \\
\hline & $\mathrm{T} 4-3.950$ & $\mathrm{~T} 4-6.120$ & $\mathrm{~T} 4-8.010$ & T4-10.430 & T4- 10.540 \\
\hline & TSH-11.01 & TSH-8.015 & TSH-6.720 & TSH-3.92 & TSH-3.64 \\
\hline \multirow[t]{3}{*}{ Case 5} & T3-0.52 & T3-0.75 & T3-1.031 & T3-0.92 & T3-0.923 \\
\hline & $\mathrm{T} 4-4.103$ & T4-6.320 & $\mathrm{T} 4-8.012$ & T4-10.540 & T4-10.234 \\
\hline & TSH-13.36 & TSH-9.012 & TSH-7.015 & TSH-3.52 & TSH-3.23 \\
\hline
\end{tabular}

$\mathrm{BL}=$ Baseline; $\mathrm{M} 1=1^{\text {st }}$ month; $\mathrm{M} 2=2^{\text {nd }}$ month; $\mathrm{M} 3=3^{\text {rd }}$ month; $\mathrm{M} 6=6^{\text {th }}$ month; TSH $=$ Thyroid stimulating hormone ( $\mu \mathrm{lU} /$ $\mathrm{mL}) ; \mathrm{T} 4=$ Total Thyroxine $(\mu \mathrm{g} / \mathrm{dL}) ; \mathrm{T} 3=$ Total Triiodothyronine $(\mathrm{ng} / \mathrm{mL})$.

Reference range: $\mathrm{TSH}(21-54$ years old $)=0.4-4.2 \mu \mathrm{lU} / \mathrm{mL} ; \mathrm{T} 4=6.09-12.23 \mu \mathrm{g} / \mathrm{dL} ; \mathrm{T} 3(11-50$ Years old $)=0.7-2.0 \mathrm{~g} / \mathrm{mL}$. 
position of the pulse in the right hand. Fire cupping diagnosis also revealed moderate stagnation of Qi with mild deficiency. Three months of acupuncture coupled with fire cupping has shown significant reduction in the TSH levels, adding to which the patient expressed subjective improvement in their quality of life and clinically reported reduction in BMI over 6 months. Further, the levels of TSH, T3 and T4 remained within the normal range even after the three months of treatment sessions (Table 2). Owing to the improvement in the thyroid functions, the medication dose was tapered at the end of the 6th month compared to the baseline. (Fig. 3 and 4).

\section{Case 2}

The second case was a 38-year-old woman who was a known case of hypothyroidism for 3 years and was under the medication- Thyronorm (Thyroxine). She also had a genetic predisposition to hypothyroidism and was overweight. Biochemical evaluation revealed elevated levels of TSH and low levels of T4 hormone, while T3 levels remained normal. Tongue diagnosis depicted spleen Qi deficiency which was marked by a mild coating with teeth marks. The pulse remained empty at the 2 nd position in the right hand whereas fire cupping diagnosis has shown the patient to have moderate stagnation with blood deficiency. Marked reduction in TSH levels and elevation in T4 levels to the normal range were observed consistently across all the evaluation points including the measurement at the end of the 3-month followup period (Table 2). The medication dose was tapered to a very minimal dose at the end of 6 months. The patient has exhibited significant reduction in weight during the entire period of observation while reporting substantial improvements in quality of life (Fig. 3 and 4).

\section{Case 3}

A 42-year-old woman with hypothyroidism for the past 7 years with genetic predisposition presented with weight gain, hair loss, lethargy, tinnitus and cold feet. She was under the medication- Thyronorm (Thyroxine) and had an elevated level of TSH, reduced T4 levels and normal T3 levels. She presented with a deep wiry pulse in the $3 \mathrm{rd}$ position as well as the 2 nd position on the right hand. Further, a pale tongue with teeth marks signifying spleen Qi deficiency was observed. Fire cupping diagnosis also showed a severe stagnation of Qi. Gradual decrease in the TSH levels and increase in T4 levels were observed across the time points, continuing post the follow-up as well (Table 2). The patient expressed improvement in daily living activities and reported the assuagement of all the symptoms within the first one month of intervention. Further reduction in medication dose, weight and other symptoms were also observed during the study period (Fig. 3 and 4).

\section{Case 4}

The patient was a 44-year-old woman and a known case of hypothyroidism for 7 years with no genetic predisposition and was under the medication- Thyronorm (Thyroxine). She presented with increased levels of TSH, reduced T4 levels

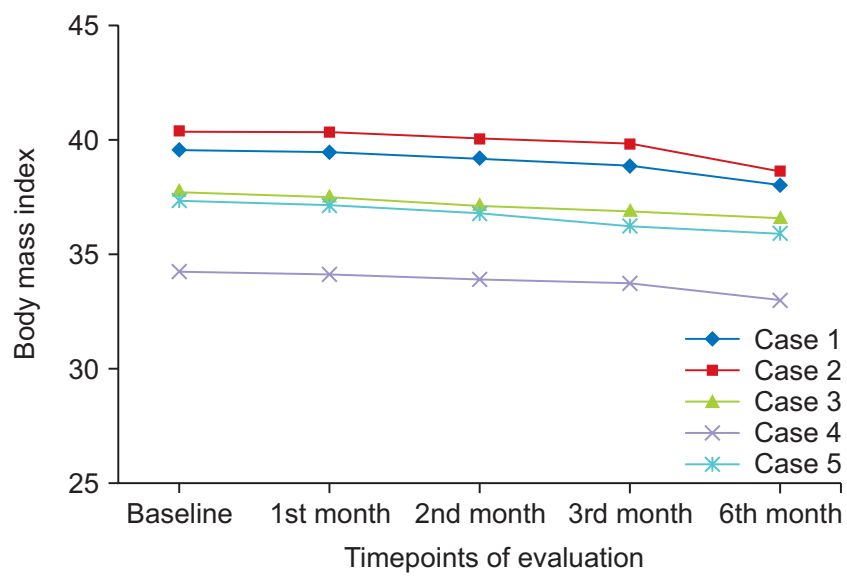

Fig. 4. Changes in body mass index from baseline through 6 months.

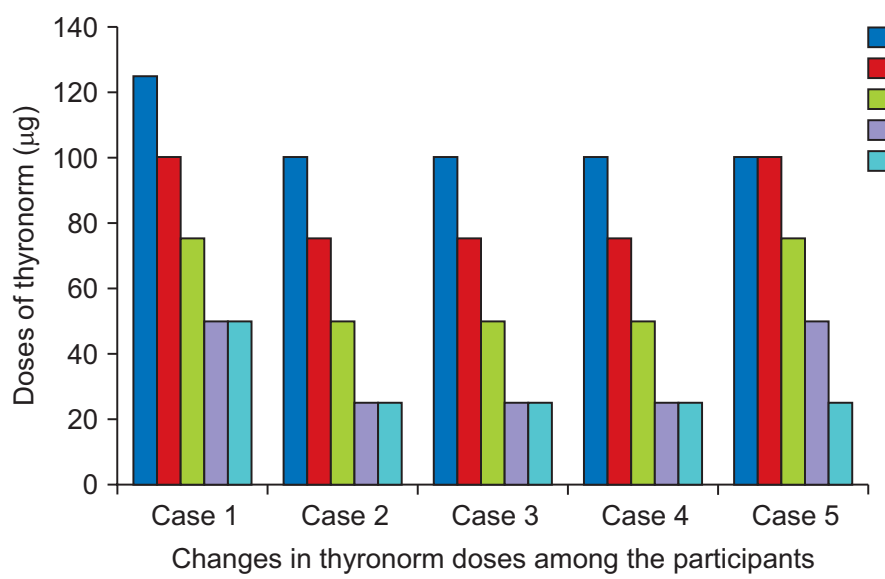

Thyronorm dose baseline

Thyronorm dose 1 month

Thyronorm dose 2 month

Thyronorm dose 3 month

Thyronorm dose 6 month

Fig. 3. Changes in thyronorm doses from baseline through 6 months. 
and normal T3 levels, supplementing which she presented with complaints of excessive intolerance towards cold, hair thinning, muscle aches and cramps even during rest. The pulse examination showed choppy wiry pulse on the 1st and 2nd position of the left hand and 1st position of the right hand. A thin white greasy coat with severe teeth marks, and cracks in the middle part of the tongue were observed in the tongue diagnosis which point to spleen Qi deficiency. Three months of acupuncture along with fire cupping had significantly reduced the TSH levels and subsequently increased the T4 levels to the normal range (Table 2). Like the previous cases, this case also showed significant reduction in the BMI and need for an escalated dose of medication as well (Fig. 3 and 4). The patient reported a reduction in thinning of hair to an appreciable extent, supplementing which also expressed improvements regarding the symptoms of muscle aches and cramps to have reduced by $70 \%$ even during rest. She was able to do her household chores with ease and comfort.

\section{Case 5}

A 41-year-old woman diagnosed with hypothyroidism for the past 4 years presented with unusual weight gain, thinning of hair and fatigue. She was under the medicationThyronorm (Thyroxine). Thyroid function tests showed elevated TSH levels and reduced T4 levels. T3 levels were within the normal range. Pulse examination showed a soft weak pulse on the right-hand $2^{\text {nd }}$ position. The tongue exhibited paleness with teeth marks and the fire cupping revealed a moderate stagnation. Like the previous cases, acupuncture and fire cupping restored the thyroid hormones back to the normal range that resulted in reduction of the Thyroxine doses and a subsequent weight reduction (Fig. 3 and 4). A reduction in thinning of hair and improved energy levels were also reported.

\section{DISCUSSION}

Our cases demonstrated significant improvement in the thyroid function, reduction in the BMI levels and medication dose following three months of acupuncture and fire cupping. To the best of our knowledge, this is the first clinical study reporting the effect of Acupuncture and Fire Cupping on hypothyroidism with a follow-up period of 3 months. Arsovka et al. earlier reported reduction in the TSH, T4 and Thyroxine peroxidase (TO) levels in a case of Hashimoto hypothyroidism post-acupuncture [8]. However, our cases were not autoimmune as their TO levels were within normal limits which makes this case series as the first report on effects of acupuncture coupled with fire cupping in treating hypothyroidism. Similar effects of acupuncture in reducing
TSH levels were reported by Zhu et al. [14]. However, this case report included only 5 sessions of acupuncture and has neither reported any follow-up prognostic features nor any changes in the dosage of medications. All the cases in the present study underwent a tapering of thyroxine doses by their treating endocrinologist to a minimal level along with reduction in BMI. The effects of acupuncture and fire cupping sustained throughout our follow-up period of 3 months and none of our patients required any dose escalation or any additional sessions for maintaining the thyroid hormone levels. Besides this, there were fewer case reports and observational studies that reported improvement in the functional status and emotional balance in hypothyroidism patients after acupuncture [15-17].

The reduction in symptoms, BMI and sustained thyroid hormone levels are indicative of balancing effects of acupuncture across the energy meridians. Similar effects have been elicited by a study conducted on 27 female participants who had subclinical hypothyroidism, where acupuncture has shown to improve the quality of life and balance the TSH levels [18]. As per TCM, a balance between Qi, Yin and Yang in the spleen, heart and kidney are paramount factors in the approach to achieve better results through acupuncture. Our case series report the efficacy of combining both fire cupping and acupuncture, as cupping has been suspected to precipitate the blood circulation and alleviate blood stasis in the body [19].

The present study reiterates the efficacy of acupuncture in the management of hypothyroidism. Acupuncture and cupping therapy have not only shown to restore the normal physiological level of thyroid hormones but also reduce the need for medication in treating hypothyroidism, which is reported as a greater challenge in the management of hypothyroidism [4]. The results of this study warrant future randomized control trials to explore the possibility of utilizing acupuncture and cupping as an adjuvant in the management of hypothyroidism.

\section{AUTHORS' CONTRIBUTIONS}

Conceptualization: PMK, NJ; Data curation: PMK, NJ; Formal analysis: PMK; Investigation: PMK, NJ; Methodology: PMK, NJ; Project administration: NJ; Resources: NJ; Supervision: PMK, NJ; Visualization: PMK, NJ; Writing original draft: PMK; Writing - review \& editing: PMK, NJ.

\section{CONFLICT OF INTEREST}

The authors declare no conflict of interest. 


\section{ORCID}

Pradeep M. K. Nair,

https://orcid.org/0000-0002-5163-8309

Naga Jyothi, https://orcid.org/0000-0001-8100-4801

\section{REFERENCES}

1. Hollowell JG, Staehling NW, Flanders WD, Hannon WH, Gunter EW, Spencer CA, et al. Serum TSH, T(4), and thyroid antibodies in the United States population (1988 to 1994): National Health and Nutrition Examination Survey (NHANES III). J Clin Endocrinol Metab 2002;87:489-99.

2. Bagcchi S. Hypothyroidism in India: more to be done. Lancet Diabetes Endocrinol 2014;2:778.

3. Chaker L, Bianco AC, Jonklaas J, Peeters RP. Hypothyroidism. Lancet 2017;390:1550-62.

4. Kansagra SM, McCudden CR, Willis MS. The challenges and complexities of thyroid hormone replacement. Lab Med 2010;41:338-48.

5. Midgley JEM, Toft AD, Larisch R, Dietrich JW, Hoermann R. Time for a reassessment of the treatment of hypothyroidism. BMC Endocr Disord 2019;19:37.

6. Onumah BM. Alternative and complementary treatment of thyroid disorders. In: Wartofsky L, Van Nostrand D, eds. Thyroid Cancer: A Comprehensive Guide to Clinical Management, 3rd ed. New York: Springer, 2016:759-65.

7. Aboushanab TS, AlSanad S. Cupping therapy: an overview from a modern medicine perspective. J Acupunct Meridian Stud 2018;11:83-7.

8. Arsovska B, Zhu J, Kozovska K. Case report: acupuncture treatment for hypothyroidism. Imp J Interdiscip Res 2016;2:184-7.

9. Maclean W, Lyttleton J. Clinical Handbook of Internal
Medicine: The Treatment of Disease with Traditional Chinese Medicine. Vol. 3, Qi, Blood, Fluid and Channel Disorders. London: Pangolin Press, 2010.

10. Cheng FK. An overview of the contribution of acupuncture to thyroid disorders. J Integr Med 2018;16:375-83.

11. Kim H, Moon SY, Han K, Lee JH, Im JH, Kim S, et al. Treatment of hypothyroidism using Korean medicine: 2 case reports. Medicine (Baltimore) 2020;99:e19737.

12. Gao LW. Practical Cupping Therapy. Beijing: Academy Press, 2004.

13. Ansorge R. Cupping therapy. Available at: https://www.webmd. com/balance/guide/cupping-therapy [Date accessed: March 18, 2021]

14. Zhu J, Arsovska B, Kozovska K. Case report: acupuncture treatment in male patient with primary hypothyroidism. Int J Res Granthaalayah 2019;7:217-20.

15. Zhou J. Clinical curative effect explorer of warm acupuncture in treatment of adult thyroid hypofunctions. China Mod Med 2013;20:139-40, 142.

16. Arsovska B, Zhu J. Thyroid disorder: treatment with acupuncture. Int J Sci Rep 2017;3:227-9.

17. Zhao Y, Wang X, Zhao X, Zhang L. [Effect of acupuncture on hypothyroidism: a report of 26 cases]. Shanghai J Acupunct Moxibustion 2005;24:25-6. Chinese.

18. Luzina KÉ, Luzina LL, Vasilenko AM. [The influence of acupuncture on the quality of life and the level of thyroidstimulating hormone in patients presenting with subclinical hypothyroidism]. Vopr Kurortol Fizioter Lech Fiz Kult 2011;(5): 29-33. Russian.

19. Tagil SM, Celik HT, Ciftci S, Kazanci FH, Arslan M, Erdamar $\mathrm{N}$, et al. Wet-cupping removes oxidants and decreases oxidative stress. Complement Ther Med 2014;22:1032-6. 